\title{
Piecewise-linear and bilinear approaches to nonlinear differential equations approximation problem of computational structural mechanics
}

\author{
Roman Leibov ${ }^{1, *}$ \\ ${ }^{1}$ Moscow state university of civil engineering, Yaroslavskoye shosse, 26, Moscow, Russia, 129337
}

\begin{abstract}
This paper presents a bilinear approach to nonlinear differential equations system approximation problem. Sometimes the nonlinear differential equations right-hand sides linearization is extremely difficult or even impossible. Then piecewise-linear approximation of nonlinear differential equations can be used. The bilinear differential equations allow to improve piecewise-linear differential equations behavior and reduce errors on the border of different linear differential equations systems areas. The matrices of bilinear differential equations system are estimated through nonlinear programming. The results of proposed approach application are presented.
\end{abstract}

\section{Linear, piecewise-linear and bilinear approximation of nonlinear differential equations}

Let's consider nonlinear differential equations [1-4].

$$
\dot{\mathbf{x}}=\mathbf{f}(\mathbf{x}, \mathbf{u})
$$

There are $\mathbf{x}-$ state vector, $\mathbf{u}-$ input vector.

The constant input vector ${ }_{s} \mathbf{u}$ determines steady-state vector of state variables ${ }_{s} \mathbf{x}$.

Nonlinear differential equations in a small neighborhood of $r$-th steady-state point are approximately described by linear differential equations [1-4].

$$
\dot{\mathbf{x}}^{n}={ }^{r} \mathbf{A}\left(\mathbf{x}^{n}-{ }_{s}^{r} \mathbf{x}^{n}\right)+{ }^{r} \mathbf{B}\left(\mathbf{u}^{n}-{ }_{s}^{r} \mathbf{u}^{n}\right)
$$

or

$$
\dot{x}_{i}^{n}={ }_{i}^{r} \mathbf{a}\left(\mathbf{x}^{n}-{ }_{s}^{r} \mathbf{x}^{n}\right)+{ }_{i}^{r} \mathbf{b}\left(\mathbf{u}^{n}-{ }_{s}^{r} \mathbf{u}^{n}\right), \quad i=1, \ldots, n .
$$

There are $\mathbf{x}^{n}$ - vector of normalized state variables, $\mathbf{u}^{n}$ - vector of normalized input variables,

$$
\begin{aligned}
& x_{i}^{n}=\frac{x_{i}}{\max \left|{ }_{s} x_{i}\right|}, \quad i=1, \ldots, n, \\
& u_{i}^{n}=\frac{u_{i}}{\left.\max \right|_{s} u_{i} \mid}, \quad i=1, \ldots, m .
\end{aligned}
$$

\footnotetext{
* Corresponding author: r leibov@,mtu-net.ru
} 


$$
\begin{aligned}
& { }^{r} \mathbf{A}=\left[\begin{array}{c}
r \\
{ }_{1} \mathbf{a} \\
\cdots \\
{ }_{n} \mathbf{a}
\end{array}\right], \\
& { }^{r} \mathbf{B}=\left[\begin{array}{c}
{ }_{1}^{r} \mathbf{b} \\
\cdots \\
{ }_{n} \mathbf{b}
\end{array}\right]
\end{aligned}
$$

are corresponding linear differential equations system matrices.

Also nonlinear differential equations in a small neighborhood of $r$-th steady-state point are approximately described by bilinear and quadratic differential equations [3], [4].

$$
\begin{gathered}
\dot{x}_{i}^{n}=\left[{ }_{i}^{r} \mathbf{a}{ }_{i}^{r} \mathbf{b}\right]\left[\begin{array}{c}
\mathbf{x}^{n}-{ }_{s}^{r} \mathbf{x}^{n} \\
\mathbf{u}^{n}-{ }_{s}^{r} \mathbf{u}^{n}
\end{array}\right]+\left[\begin{array}{c}
\mathbf{x}^{n}-{ }_{s}^{r} \mathbf{x}^{n} \\
\mathbf{u}^{n}-{ }_{s}^{r} \mathbf{u}^{n}
\end{array}{ }^{r} \boldsymbol{\Gamma}\left[\begin{array}{c}
\mathbf{x}^{n}-{ }_{s}^{r} \mathbf{x}^{n} \\
\mathbf{u}^{n}-{ }_{s}^{r} \mathbf{u}^{n}
\end{array}\right], \quad i=1, \ldots, n,\right. \\
\dot{x}_{i}^{n}={ }_{i}^{r} \mathbf{a}\left(\mathbf{x}^{n}-{ }_{s}^{r} \mathbf{x}^{n}\right)+{ }_{i}^{r} \mathbf{b}\left(\mathbf{u}^{n}-{ }_{s}^{r} \mathbf{u}^{n}\right)+ \\
\left(\mathbf{x}^{n}-{ }_{s}^{r} \mathbf{x}^{n}\right)^{T}{ }_{i}^{r} \boldsymbol{\Gamma}^{\mathbf{x}}\left(\mathbf{x}^{n}-{ }_{s}^{r} \mathbf{x}^{n}\right)+\left(\mathbf{u}^{n}-{ }_{s}^{r} \mathbf{u}^{n}\right)^{T}{ }_{i}^{r} \boldsymbol{\Gamma}^{\mathbf{u}}\left(\mathbf{u}^{n}-{ }_{s}^{r} \mathbf{u}^{n}\right), \quad i=1, \ldots, n,
\end{gathered}
$$

where

$$
\begin{aligned}
{ }_{i}^{r} \boldsymbol{\Gamma} & =\left[\begin{array}{cccc}
{ }_{i}^{r} \gamma_{11} & { }_{i}^{r} \gamma_{12} & \ldots & { }_{i}^{r} \gamma_{1, n+m} \\
0 & { }_{i}^{r} \gamma_{22} & \cdots & { }_{i}^{r} \gamma_{2, n+m} \\
\ldots & \ldots & \ldots & \ldots \\
0 & 0 & \ldots & { }_{i}^{r} \gamma_{n+m, n+m}
\end{array}\right], \quad i=1, \ldots, n, \\
{ }_{i}^{r} \boldsymbol{\Gamma}^{\mathbf{x}} & =\left[\begin{array}{cccc}
{ }_{i}^{r} \gamma_{11}^{\mathbf{x}} & { }_{i}^{r} \gamma_{12}^{\mathbf{x}} & \ldots & { }_{i}^{r} \gamma_{1 n}^{\mathbf{x}} \\
0 & { }_{i}^{r} \gamma_{22}^{\mathbf{x}} & \ldots & { }_{i}^{r} \gamma_{2 n}^{\mathbf{x}} \\
\ldots & \ldots & \ldots & \ldots \\
0 & 0 & \ldots & { }_{i}^{r} \gamma_{n n}^{\mathbf{x}}
\end{array}\right], \quad i=1, \ldots, n, \\
{ }_{i}^{r} \boldsymbol{\Gamma}^{\mathbf{u}} & =\left[\begin{array}{cccc}
{ }_{i}^{r} \gamma_{11}^{\mathbf{u}} & { }_{i}^{r} \gamma_{12}^{\mathrm{u}} & \ldots & { }_{i}^{r} \gamma_{1 m}^{\mathbf{u}} \\
0 & { }_{i}^{r} \gamma_{22}^{\mathrm{u}} & \ldots & { }_{i}^{r} \gamma_{2 m}^{\mathbf{u}} \\
\ldots & \ldots & \ldots & \ldots \\
0 & 0 & \ldots & { }_{i}^{r} \gamma_{m m}^{\mathbf{u}}
\end{array}\right], \quad i=1, \ldots, n .
\end{aligned}
$$

The derivatives of right-hand side of equation (11) are

$$
\begin{gathered}
\frac{d\left[{ }_{i}^{r} \mathbf{a}\left(\mathbf{x}^{n}-{ }_{s}^{r} \mathbf{x}^{n}\right)\right]}{d\left(\mathbf{x}^{n}\right)^{T}}+\frac{d\left[{ }_{i}^{r} \mathbf{b}\left(\mathbf{u}^{n}-{ }_{s}^{r} \mathbf{u}^{n}\right)\right]}{d\left(\mathbf{x}^{n}\right)^{T}}+\frac{d\left[\left(\mathbf{x}^{n}-{ }_{s}^{r} \mathbf{x}^{n}\right)^{T}{ }_{i}^{r} \boldsymbol{\Gamma}^{\mathbf{x}}\left(\mathbf{x}^{n}-{ }_{s}^{r} \mathbf{x}^{n}\right)\right]}{d\left(\mathbf{x}^{n}\right)^{T}}+\frac{d\left[\left(\mathbf{u}^{n}-{ }_{s}^{r} \mathbf{u}^{n}\right)^{T}{ }_{i}^{r} \boldsymbol{\Gamma}^{\mathbf{u}}\left(\mathbf{u}^{n}-{ }_{s}^{r} \mathbf{u}^{n}\right)\right]}{d\left(\mathbf{x}^{n}\right)^{T}}= \\
\frac{d\left[{ }_{i}^{r} \mathbf{a}\left(\mathbf{x}^{n}-{ }_{s}^{r} \mathbf{x}^{n}\right)\right]}{d\left(\mathbf{u}^{n}\right)^{T}}+\frac{\left.d\left[\mathbf{x}^{n}-{ }_{s}^{r} \mathbf{x}_{i}^{n}\right)^{T} \mathbf{b}\left(\mathbf{u}^{n}-{ }_{i}^{r} \boldsymbol{\Gamma}^{\mathbf{x}^{T}}+\mathbf{u}_{i}^{n}\right)\right]}{d\left(\mathbf{\Gamma}^{n}\right), \quad i=1, \ldots, n,}+\frac{\left.d\left[\left(\mathbf{x}^{n}\right)^{r}{ }_{s}^{r} \mathbf{x}^{n}\right)_{i}^{T} \boldsymbol{\Gamma}^{\mathbf{x}}\left(\mathbf{x}^{n}-{ }_{s}^{r} \mathbf{x}^{n}\right)\right]}{d\left(\mathbf{u}^{n}\right)^{T}}+\frac{d\left[\left(\mathbf{u}^{n}-{ }_{s}^{r} \mathbf{u}^{n}\right)_{i}^{T}{ }_{i}^{r} \boldsymbol{\Gamma}^{\mathbf{u}}\left(\mathbf{u}^{n}-{ }_{s}^{r} \mathbf{u}^{n}\right)\right]}{d\left(\mathbf{u}^{n}\right)^{T}}= \\
{ }_{i}^{r} \mathbf{b}+\left(\mathbf{u}^{n}-{ }_{s}^{r} \mathbf{u}^{n}\right)^{T}\left({ }_{i}^{r} \boldsymbol{\Gamma}^{\mathbf{u}^{T}}+{ }_{i}^{r} \boldsymbol{\Gamma}^{\mathbf{u}}\right), \quad i=1, \ldots, n .
\end{gathered}
$$

The values of these derivatives at

$$
\left[\begin{array}{l}
{ }_{s}^{r} \mathbf{x}^{n} \\
{ }_{s}^{r} \mathbf{u}^{n}
\end{array}\right]
$$

are ${ }_{i}^{r} \mathbf{a},{ }_{i}^{r} \mathbf{b}, i=1, \ldots, n$ and at

$$
\left[\begin{array}{c}
{ }_{s}^{r-1} \mathbf{x}^{n} \\
{ }_{r-1} \mathbf{u}^{n}
\end{array}\right]
$$

these derivatives are the rows of $(r-1)$-th steady-state point linear differential equations system matrices. 


$$
\begin{aligned}
{ }_{i}^{r-1} \mathbf{a}={ }_{i}^{r} \mathbf{a}+\left({ }_{s}^{r-1} \mathbf{x}^{n}-{ }_{s}^{r} \mathbf{x}^{n}\right)^{T}\left({ }_{i}^{r} \boldsymbol{\Gamma}^{\mathbf{x}^{T}}+{ }_{i}^{r} \boldsymbol{\Gamma}^{\mathbf{x}}\right), \quad i=1, \ldots, n, \\
{ }_{i}^{r-1} \mathbf{b}={ }_{i}^{r} \mathbf{b}+\left({ }_{s}^{r-1} \mathbf{u}^{n}-{ }_{s}^{r} \mathbf{u}^{n}\right)^{T}\left({ }_{i}^{r} \boldsymbol{\Gamma}^{\mathbf{u}^{T}}+{ }_{i}^{r} \boldsymbol{\Gamma}^{\mathbf{u}}\right), \quad i=1, \ldots, n .
\end{aligned}
$$

\section{Quadratic differential equations system matrices estimation}

Nonlinear differential equations time responses ${ }^{\mathrm{NL}} \mathbf{u}^{n}\left(t_{k}\right), k=0, \ldots, N-1,{ }^{\mathrm{NL}} \mathbf{x}^{n}\left(t_{k}\right)$, $k=0, \ldots, N$, where $t_{k+1}=t_{k}+\Delta t, k=0, \ldots, N-1$, are used for quadratic differential equations system matrices estimation. The ${ }^{\mathrm{NL}} \mathbf{u}^{n}\left(t_{k}\right), \quad k=0, \ldots, N-1$ stepwise time responses cross the border of $r$-th and $(r-1)$-th linear differential equations systems application areas.

Quadratic differential equations system matrices estimation can be reduced to nonlinear programming problem

$$
\begin{aligned}
& { }_{1}^{r} \boldsymbol{\Gamma}^{\mathbf{x}}, \ldots,{ }_{n}^{r} \boldsymbol{\Gamma}^{\mathbf{x}},{ }_{1}^{r} \boldsymbol{\Gamma}^{\mathbf{u}}, \ldots,{ }_{n}^{r} \boldsymbol{\Gamma}^{\mathbf{u}}: \min \left\{\sqrt{\frac{\sum_{k=1}^{N}\left[\mathbf{x}^{n}\left(t_{k}\right)-{ }^{\mathrm{NL}} \mathbf{x}^{n}\left(t_{k}\right)\right]^{T}{ }_{\mathbf{x}} \mathbf{W}\left(t_{k}\right)\left[\mathbf{x}^{n}\left(t_{k}\right)-{ }^{\mathrm{NL}} \mathbf{x}^{n}\left(t_{k}\right)\right]}{\sum_{k=1}^{N}\left[{ }^{\mathrm{NL}} \mathbf{x}^{n}\left(t_{k}\right)-{ }^{\mathrm{NL}} \mathbf{x}^{n}\left(t_{0}\right)\right]^{T} \mathbf{W}\left(t_{k}\right)\left[{ }^{\mathrm{NL}} \mathbf{x}^{n}\left(t_{k}\right)-{ }^{\mathrm{NL}} \mathbf{x}^{n}\left(t_{0}\right)\right]}}\right. \\
& \dot{x}_{i}^{n}\left(t_{k}\right)={ }_{i}^{r} \mathbf{a}\left[\mathbf{x}^{n}\left(t_{k}\right)-{ }_{s}^{r} \mathbf{x}^{n}\right]+{ }_{i}^{r} \mathbf{b}\left[{ }^{\mathrm{NL}} \mathbf{u}^{n}\left(t_{k}\right)-{ }_{s}^{r} \mathbf{u}^{n}\right]+ \\
& \left.\begin{array}{c}
{\left[\mathbf{x}^{n}\left(t_{k}\right){ }_{s}^{r} \mathbf{x}^{n}\right]_{i}^{T}{ }_{i}^{r} \boldsymbol{\Gamma}^{\mathbf{x}}\left[\mathbf{x}^{n}\left(t_{k}\right){ }_{s}^{r} \mathbf{x}^{n}\right]+\left[{ }^{\mathrm{NL}} \mathbf{u}^{n}\left(t_{k}\right)-{ }_{s}^{r} \mathbf{u}^{n}\right]^{T}{ }_{i} \boldsymbol{\Gamma}^{\mathrm{u}}\left[{ }^{\mathrm{NL}} \mathbf{u}^{n}\left(t_{k}\right)-{ }_{s}^{r} \mathbf{u}^{n}\right],} \\
x_{i}^{n}\left(t_{k+1}\right)=x_{i}^{n}\left(t_{k}\right)+\Delta t \dot{x}_{i}^{n}\left(t_{k}\right), \quad i=1, \ldots, n, \quad k=0,1, \ldots, N-1
\end{array}\right\} .
\end{aligned}
$$

There are ${ }_{\mathbf{x}} \mathbf{W}\left(t_{k}\right), k=1, \ldots, N$ positive definite diagonal weight matrices, ${ }^{r} \mathbf{A},{ }^{r} \mathbf{B}$ are considered known, the initial values of quadratic differential equations system matrices ${ }_{i}^{r} \boldsymbol{\Gamma}^{\mathbf{x}}=\mathbf{0},{ }_{i}^{r} \boldsymbol{\Gamma}^{\mathrm{u}}=\mathbf{0}, i=1, \ldots, n$, and $\mathbf{x}^{n}\left(t_{0}\right)={ }^{\mathrm{NL}} \mathbf{x}^{n}\left(t_{0}\right)$.

\section{Quadratic differential equations system matrices estimation example}

In this example $m=4, n=5$, vectors of normalized steady-state values of input and state variables are

$$
\begin{gathered}
{ }_{s}^{r} \mathbf{u}^{n}=[1,0.4515,0,0]^{T}, \\
{ }_{s}^{r} \mathbf{x}^{n}=[1,1,1,1,1]^{T}, \\
{ }_{s}^{r-1} \mathbf{x}^{r} \mathbf{u}^{n}=[0.9442,0.4441,-0.0893,0]^{T}, \\
\left.{ }_{s}^{r} .9741,0.9834,0.9656,0.9783,0.9790\right]^{T},
\end{gathered}
$$

linear differential equations system matrices

corresponding eigenvalues

$$
{ }^{r} \mathbf{A}=\left[\begin{array}{ccccc}
-1.8208 & 1.1770 & 0 & 0 & 0 \\
0.0697 & -1.6507 & 0 & 0 & 0 \\
9.7385 & 11.5191 & -20.1171 & -13.1868 & 0 \\
9.6711 & 3.5115 & -5.5917 & -19.3568 & 0 \\
2.9867 & -6.8163 & -3.4159 & -3.1171 & -30.0422
\end{array}\right],
$$

$$
{ }^{r} \lambda_{1}=-1.4370,{ }^{r} \lambda_{2}=-2.0345,{ }^{r} \lambda_{3}=-11.1415,{ }^{r} \lambda_{4}=-28.3324,{ }^{r} \lambda_{5}=-30.0422,
$$




$$
{ }^{r} \mathbf{B}=\left[\begin{array}{cccc}
0.6093 & 0.8201 & -0.1387 & 0.1788 \\
0.4454 & 0.5736 & -0.0387 & -0.2204 \\
10.8333 & -40.9063 & 2.6207 & 1.7204 \\
7.1854 & -39.0036 & 2.1503 & 0.5060 \\
16.0831 & 3.7726 & -0.8053 & -1.2318
\end{array}\right]
$$

At first quadratic differential equations system matrices for two differential equations are estimated.

$$
\begin{gathered}
{ }_{1}^{r} \Gamma^{\mathbf{x}}=\left[\begin{array}{cc}
-0.0162 & -0.0181 \\
0 & 0.0278
\end{array}\right], \\
{ }_{2}^{r} \Gamma^{\mathbf{x}}=\left[\begin{array}{ccc}
-0.1089 & 0.0133 \\
0 & -0.0417
\end{array}\right], \\
{ }_{1}^{r} \boldsymbol{\Gamma}^{\mathrm{u}}=\left[\begin{array}{cccc}
-0.0319 & -0.1280 & 0.0823 & -0.2076 \\
0 & 0.1117 & 0.0651 & 0.1743 \\
0 & 0 & 0.0041 & 0.1553 \\
0 & 0 & 0 & -0.4165
\end{array}\right], \\
{ }_{2}^{r} \boldsymbol{\Gamma}^{\mathrm{u}}=\left[\begin{array}{cccc}
-0.2445 & 0.0548 & 0.3299 & -0.1507 \\
0 & -0.0267 & 0.0975 & -0.0024 \\
0 & 0 & -0.0881 & -0.2008 \\
0 & 0 & 0 & 0.0639
\end{array}\right] .
\end{gathered}
$$

Then quadratic differential equations system matrices for 3-rd and 4-th differential equations are estimated considering the matrices ${ }_{1}^{r} \Gamma^{\mathrm{x}},{ }_{2}^{r} \Gamma^{\mathrm{x}},{ }_{1}^{r} \Gamma^{\mathrm{u}},{ }_{2}^{r} \boldsymbol{\Gamma}^{\mathrm{u}}$.

$$
\begin{aligned}
&{ }_{3}^{r} \Gamma^{\mathbf{x}}=\left[\begin{array}{cccc}
-1.2607 & 0.2927 & -1.6746 & -0.8985 \\
0 & 0.0186 & -0.2216 & -0.3720 \\
0 & 0 & 1.9339 & -0.5949 \\
0 & 0 & 0 & 2.4800
\end{array}\right], \\
&{ }_{4}^{r} \Gamma^{\mathbf{x}}=\left[\begin{array}{cccc}
0.3141 & 0.2177 & 0.0014 & -2.5816 \\
0 & 0.7004 & 0.9653 & -0.9625 \\
0 & 0 & -1.1875 & -0.6546 \\
0 & 0 & 0 & -0.7394
\end{array}\right], \\
&{ }_{3}^{r} \Gamma^{\mathbf{u}}=\left[\begin{array}{cccc}
-2.6416 & 0.1334 & -2.2222 & -1.0052 \\
0 & 0.4733 & 0.1910 & -2.4470 \\
0 & 0 & -0.7613 & 0.1514 \\
0 & 0 & 0 & -1.5057
\end{array}\right], \\
&{ }_{4}^{r} \boldsymbol{\Gamma}^{\mathbf{u}}=\left[\begin{array}{cccc}
0.6351 & 0.2250 & -0.5388 & 0.1228 \\
0 & -3.3969 & -0.8555 & -0.2930 \\
0 & 0 & -1.4348 & -1.3817 \\
0 & 0 & 0 & 0.3208
\end{array}\right] .
\end{aligned}
$$

At last quadratic differential equations system matrices for 5-th differential equations are estimated considering the matrices ${ }_{1}^{r} \boldsymbol{\Gamma}^{\mathrm{x}},{ }_{2}^{r} \boldsymbol{\Gamma}^{\mathrm{x}},{ }_{1}^{r} \boldsymbol{\Gamma}^{\mathrm{u}},{ }_{2}^{r} \boldsymbol{\Gamma}^{\mathrm{u}},{ }_{3}^{r} \boldsymbol{\Gamma}^{\mathrm{x}},{ }_{4}^{r} \boldsymbol{\Gamma}^{\mathrm{x}},{ }_{3}^{r} \boldsymbol{\Gamma}^{\mathrm{u}},{ }_{4}^{r} \boldsymbol{\Gamma}^{\mathrm{u}}$.

$$
\begin{gathered}
{ }_{5}^{r} \Gamma^{\mathrm{x}}=\left[\begin{array}{ccccc}
0.0764 & -3.4153 & -0.4633 & -0.6979 & 0.2337 \\
0 & 0.4479 & -1.2369 & 0.8377 & 1.3469 \\
0 & 0 & -0.9704 & -0.8798 & -0.5260 \\
0 & 0 & 0 & -1.0099 & -1.5035 \\
0 & 0 & 0 & 0 & -0.1620
\end{array}\right], \\
{ }_{5}^{r} \Gamma^{\mathrm{u}}=\left[\begin{array}{cccc}
-1.5124 & 0.9865 & 0.3452 & -2.4686 \\
0 & 1.2401 & 0.2039 & 0.5581 \\
0 & 0 & 0.3440 & -1.4374 \\
0 & 0 & 0 & 0.9207
\end{array}\right] .
\end{gathered}
$$

There are $k=1, \ldots, 2001, \Delta t=0.025$, and the weight matrices are identity matrices. Since matrix ${ }^{r-1} \mathbf{A}$ is calculated through quadratic differential equations system matrices, 
additional constraints are used in order to provide matrix ${ }^{r-1} \mathbf{A}$ real and different eigenvalues.

$$
{ }^{r-1} \mathbf{A}=\left[\begin{array}{ccccc}
-1.8197 & 1.1765 & 0 & 0 & 0 \\
0.0751 & -1.6497 & 0 & 0 & 0 \\
9.8760 & 11.5266 & -20.1902 & -13.2445 & 0 \\
9.7072 & 3.4703 & -5.5119 & -19.2194 & 0 \\
3.0656 & -6.7466 & -3.2865 & -3.0073 & -30.0131
\end{array}\right]
$$

corresponding eigenvalues

$$
\begin{gathered}
{ }^{r-1} \lambda_{1}=-1.4255,{ }^{r-1} \lambda_{2}=-2.0439,{ }^{r-1} \lambda_{3}=-11.1469,{ }^{r-1} \lambda_{4}=-28.2627,{ }^{r-1} \lambda_{5}=-30.0131, \\
{ }^{r-1} \mathbf{B}=\left[\begin{array}{cccc}
0.6065 & 0.8198 & -0.1445 & 0.1752 \\
0.4428 & 0.5622 & -0.0421 & -0.1941 \\
11.3250 & -40.9378 & 2.8790 & 1.7846 \\
7.1611 & -38.8895 & 2.4429 & 0.6247 \\
16.2135 & 3.6811 & -0.8875 & -0.6247
\end{array}\right] .
\end{gathered}
$$

Some results of proposed method application are presented at Fig. 1, 2. The piecewiselinear differential equations system state variable derivative ${ }^{\mathrm{PL}} \dot{x}_{5}^{n}$ time responce surge on the border of two linear differential equations systems application areas is much less if matrix ${ }^{r-1} \mathbf{A}$ is calculated through quadratic differential equations system matrices.

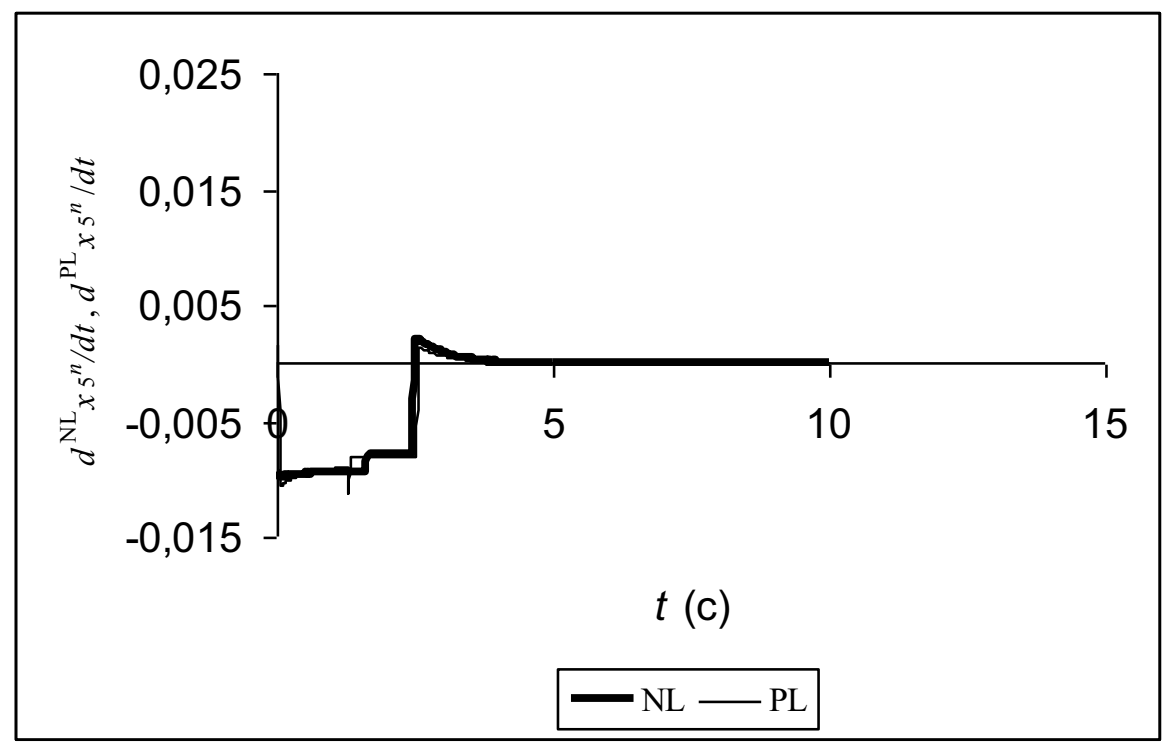

Fig. 1. Nonlinear and piecewise-linear differential equations systems state variables ${ }^{\mathrm{NL}} x_{5}^{n}$ and ${ }^{\mathrm{PL}} x_{5}^{n}$ derivatives ${ }^{\mathrm{NL}} \dot{x}_{5}^{n}$ and ${ }^{\mathrm{PL}} \dot{x}_{5}^{n}$ time responces $\left({ }^{r-1} \mathbf{A}\right.$ is calculated through quadratic differential equations system matrices). 


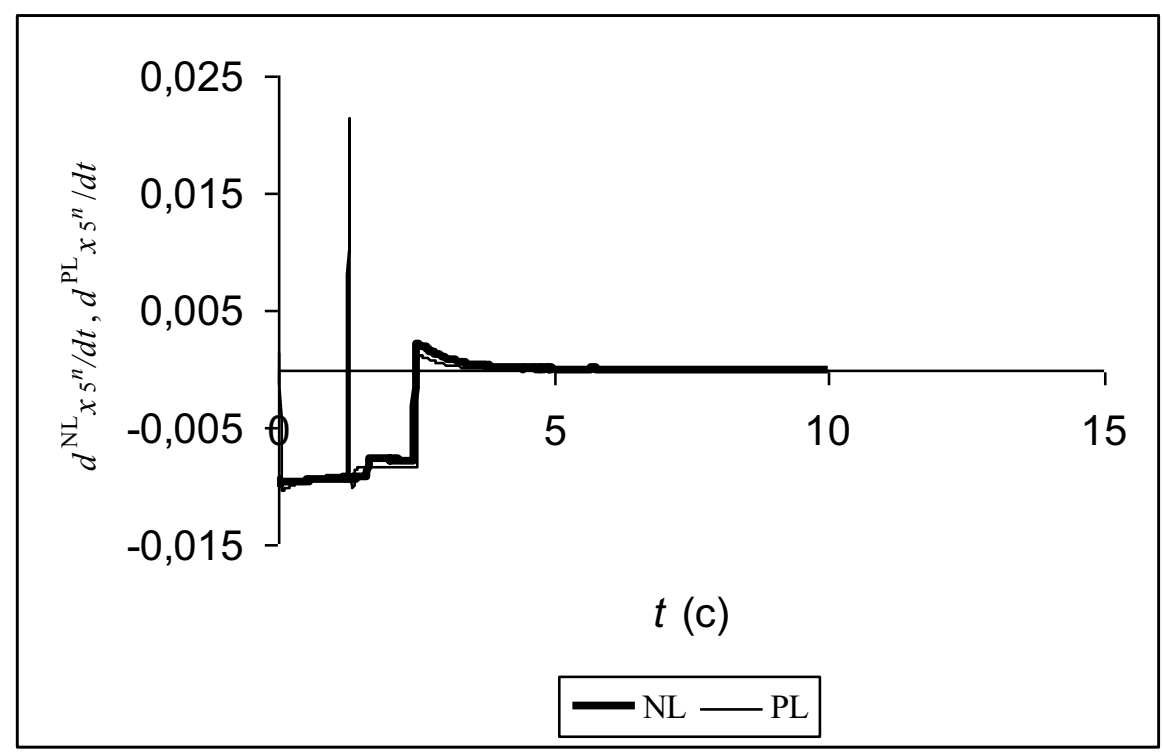

Fig. 2. Nonlinear and piecewise-linear differential equations systems state variables ${ }^{\mathrm{NL}} x_{5}^{n}$ and ${ }^{\mathrm{PL}} x_{5}^{n}$ derivatives ${ }^{\mathrm{NL}} \dot{x}_{5}^{n}$ and ${ }^{\mathrm{PL}} \dot{x}_{5}^{n}$ time responces.

\section{References}

1. R.L. Leibov, IJCCSE 8, 1, 96-107 (2012)

2. R.L. Leibov, IJCCSE 9, 4, 186-198 (2013)

3. R.L. Leibov, IJCCSE 11, 2, 107-130 (2015)

4. R.L. Leibov, IJCCSE 12, 3, 40-57 (2016) 